\title{
Modified Orbitozygomatic Approach without Orbital Roof Removal for Middle Fossa Lesions
}

\author{
Ramiro López-Elizalde, M.D., ${ }^{1,2}$ Edgar Robledo-Moreno, M.D.,' Gabriel O’Shea-Cuevas, M.D., ${ }^{3}$ \\ Esmeralda Matute-Villaseñor, M.D., ${ }^{4}$ Álvaro Campero, M.D., ${ }^{5}$ Marisol Godínez-Rubí, M.D. ${ }^{1,6}$ \\ Neurosurgery Service,' Valentín Gómez Farías General Hospital, Institute of Security and Social Services for State Workers, Guadalajara, \\ Jalisco, México \\ Neurosurgery Service, ${ }^{2}$ Hospital Civil de Guadalajara "Dr. Juan I. Menchaca", Guadalajara, Jalisco, México \\ Toluca Medical Center, Metepec, Estado de México, México \\ Institute of Neurosciences, ${ }^{4}$ University of Guadalajara, Guadalajara, Jalisco, México \\ Neurosurgery Service, ${ }^{5}$ Padilla Hospital, Tucuman, Argentina \\ Laboratory of Pathology Research, ${ }^{6}$ Department of Microbiology and Pathology, University Center of Health Sciences, University of Guadala- \\ jara, Guadalajara, Jalisco, México
}

Objective : The purpose of the present study was to describe an OrBitoZygomatic (OBZ) surgical variant that implies the drilling of the orbital roof and lateral wall of the orbit without orbitotomy.

Methods : Design : cross-sectional study. Between January 2010 and December 2014, 18 patients with middle fossa lesions underwent the previously mentioned OBZ surgical variant. Gender, age, histopathological diagnosis, complications, and percentage of resection were registered. The detailed surgical technique is described.

Results : Of the 18 cases listed in the study, nine were males and nine females. Seventeen cases (94.5\%) were diagnosed as primary tumoral lesions, one case (5.5\%) presented with metastasis of a carcinoma, and an additional one had a fibrous dysplasia. Age ranged between 27 and 73 years. Early complications were developed in four cases, but all of these were completely resolved. None developed enophthalmos.

Conclusion : The present study illustrates a novel surgical OBZ approach that allows for the performance of a simpler and faster procedure with fewer complications, and without increasing surgical time or cerebral manipulation, for reaching lesions of the middle fossa. Thorough knowledge of the anatomy and surgical technique is essential for successful completion of the procedure.

Key Words : Skull base · Orbit · Zygoma · Neurosurgery · Craniotomy · Cranial fossa, Middle.

\section{INTRODUCTION}

The frontotemporal OrBitoZygomatic (OBZ) approach is considered one of the main accesses and one of the most ap- plied procedures in skull base surgery ${ }^{2,10,14,22,24)}$. Hakuba first described the OBZ access in 1986, which resulted from small changes in the fronto-temporo-sphenoidal craniotomy ${ }^{11}$. Currently, OBZ approach comprises a group of surgical tech-

\footnotetext{
- Received : July 7, 2017 •Revised : October 10, 2017 •Accepted : November 3, 2017

- Address for reprints : Marisol Godínez-Rubí, M.D.

Neurosurgery Service, Valentín Gómez Farías General Hospital, Institute of Security and Social Services for State Workers, Sierra Mojada No 950, Edificio 0, Planta Baja. Colonia Independencia, C.P. 44340, Guadalajara, Jalisco, México

Tel : +52 $13310585200-33634$, Fax : +52 3338360660, E-mail : jmarisol.godinezrubi@gmail.com
}

This is an Open Access article distributed under the terms of the Creative Commons Attribution Non-Commercial License (http://creativecommons.org/licenses/by-nc/4.0) which permits unrestricted non-commercial use, distribution, and reproduction in any medium, provided the original work is properly cited. 
niques to access to skull-base injuries involving elements of the frontal, temporal, and sphenoidal regions, as well as the orbital wall and the zygomatic bone ${ }^{10,12,22)}$. OBZ craniotomy combines the advantages of the pterional and pretemporal approaches, but improves the angle of the microscope view. Hence, it provides the best view for brain injuries in the floor of the anterior and middle cranial fossa ${ }^{2,5,6,10,22)}$.

Main variants of the OBZ approach include those in which

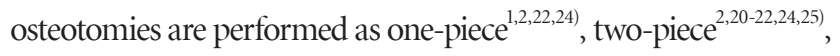
or three-piece bone flaps ${ }^{4,5,18)}$. In all of these, a craniotomy is carried out that allows resection of the frontotemporal fragment, the roof, and the lateral wall of the orbit, of the zygoma lateral surface, and of the zygomatic process of temporal bone, in one, two, or three segments, respectively ${ }^{24)}$. Each technique possesses advantages, disadvantages, and alternatives that can be tailored to the patient's characteristics and to particular lesions, enabling the surgeon to choose the best technique $^{6,10,18,22)}$.

However, all of the surgical variants have potential complications of the orbital osteotomy : the development of enophthalmos, and the functional and cosmetic defects arising from this $^{2,22,24)}$. To avoid these complications, we have developed a variant of the OBZ approach in which we avoid the orbital osteotomy, and instead perform orbital drilling. Later, we present a detailed description of an OBZ access without removing the orbital roof, taking advantage of the whole vision field and maneuverability provided by an OBZ conventional approach, but avoiding the development of complications inherent to the traditional approach.

\section{MATERIALS AND METHODS}

\section{Patients}

We performed a retrospective study including 18 patients submitted to a surgical procedure between January 2010 and December 2014, in whom a modified OBZ approach was the treatment-of-choice due to lesions of the middle cranial fossa. Patient information included tumor type, gender, age, complications, and percentage of resection. Follow-up was at least 36 months in all patients.

\section{Model of the proposed surgical technique}

The proposed surgical model was performed in two sili- cone-injected, formalin-fixed cadaveric heads to illustrate the process. The step-by-step description of the surgical technique and the relevant anatomy is documented.

\section{Surgical technique description}

\section{Patient's positioning}

The patient is placed in the decubitus supine position, with the head rotated $30-60^{\circ}$ to the side opposite the surgical incision (the degree of rotation will depend on lesion localization and extension). The neck is slightly extended. This maneuver facilitates retraction of the frontal lobe away from the orbital roof and the skull base by gravity (Fig. 1A).

\section{Skin incision}

The skin incision starts at $1 \mathrm{~cm}$ anterior to the tragus in a vertical form, begins to be curvilinear at the superior temporal line, and extends to the hemipupillary contralateral line (Fig. 1A). The superficial temporal artery, whose posterior branch is cut off, should be identified early, so that main artery trunk is moved forward along with the cutaneous flap.

\section{Elevation of the temporal flap}

The periosteum should be separated from the incision's posterior border. The frontotemporal branch of the facial nerve runs between the galea and the superficial temporalis fascia to innervate the frontalis muscle. To avoid damaging to this branch, the external superficial fascia is separated from its internal portion and reflected forward with the skin flap. The incision in the fascia starts $2 \mathrm{~cm}$ behind the posterior prominence of the zygomatic process of the frontal bone at the superior temporal line and continues inferiorly, parallel to the frontozygomatic process, toward the zygomatic arch. The deep fascia of the temporal muscle is dissected retrogradedly with a periosteum dissector or elevator, which preserves the aponeurotic blade and the neurovascular supply, minimizing the problems of postoperative atrophy (Fig. 1B). A $3 \times 1 \mathrm{~cm}$ muscle and fascia flange is maintained at the level of the superior temporal line, allowing muscle reinsertion and covering the craniotomy path at the time of surgical repair. Once disinserted, the muscle is rejected toward the malar eminence. The degree of muscle descent will depend on the localization of the lesion, the working vector that the surgeon is employing, and whether the zygomatic arch osteotomy is performed (Fig. 1C). 


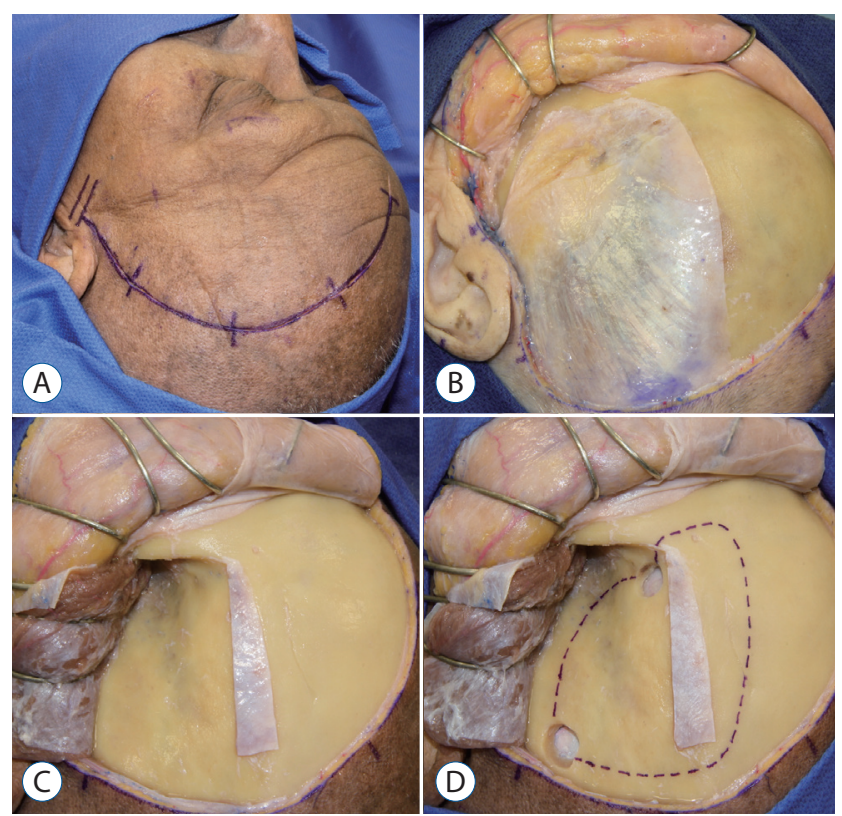

Fig. 1. A : The location of the skin incision is strategic. Thus, it respects the main trunk of the superficial temporal artery, the auriculotemporal nerve, and the main trunk of the VII cranial nerve and its frontotemporal and supraorbital branches. B : Main artery trunk of the superficial temporal artery is displaced forward along with the cutaneous flap. Later, the deep fascia of the temporal muscle is dissected retrogradedly to preserve the aponeurotic blade and the neurovascular supply. C : Once the cutaneous flap is elevated, the temporal muscle is dissected for disinsertion and rejection toward the malar eminence, leaving a muscle and fascia eyebrow of approximately $3 \times 1 \mathrm{~cm}$ at the level of the superior temporal line, as well as at the location of the primary trepan. D : Frontotemporo-sphenoidal (pterional) craniotomy model in the variant of the proposed surgical technique.

\section{Pterional craniotomy (fronto-temporo-sphenoidal)}

A trepanation is set one $\mathrm{cm}$ posterior to the frontozygomatic union, thus rendering it possible to drill additional holes along the proposed route for the bony flap, which will depend on how closely the dura mater is to the bone. We prefer to drill at the level of the sphenoid wing and the most basal part of the craniotomy, rather than to utilize the craniotome, in order to maintain dura mater integrity. The craniotomy is completed at the supraorbital notch (medial limit), ensuring that the cutting line performed flush through the orbital roof is performed with care not to enter the frontal sinus. It is important to reach the skull base and arrive as far back as possible toward the temporal fossa (Figs. 1D and 2A).

\section{Orbital cone drilling}

Later, all roof bony protrusions, the orbit lateral wall, and the sphenoid minor wing are drilled, setting the meningo-or-
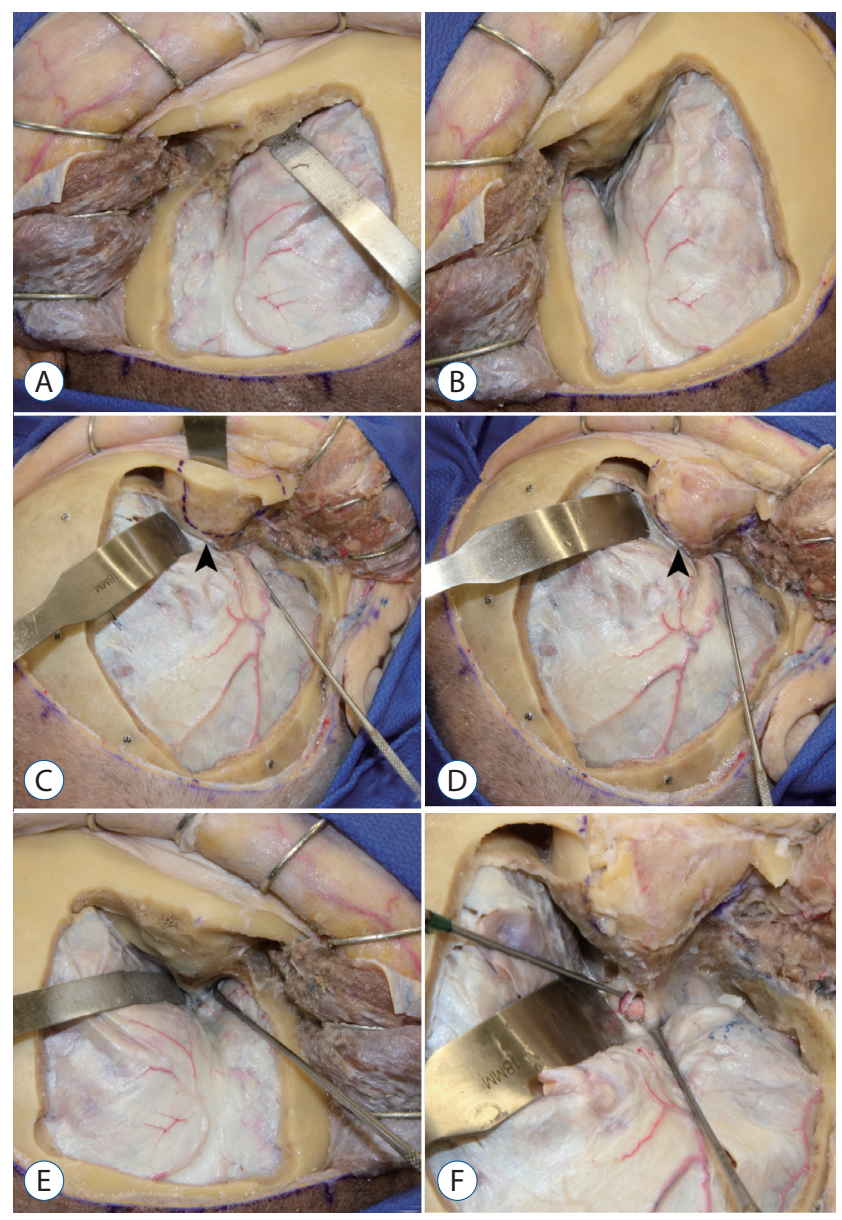

Fig. 2. A : The bony surfaces of the orbital roof and the lateral wall are observed after the pterional craniotomy, rendering it difficult to access the cranial vault. It is necessary to retract the brain to a greater degree in order to achieve better visualization of the skull base when the orbital roof is not drilled. $B$ : In this image, the proposed technique is shown: an OBZ approach without orbitotomy but with the drilling of orbital roof and lateral wall. It can be observed that that drilling of all roof bony protrusions enlarges the access field to the cranial fossa. $C$ : The same procedure as in (B) was performed. The dashed lines indicate the site where the osteotomy would be performed in a conventional OBZ approach. D : Conventional OBZ approach after orbitotomy with exposure of periorbital soft tissues. When comparing (B) and (C) with (D), it may be observed that, with sufficient drilling, there is no need to remove the orbital roof and wall, unless the pathology involves the orbit itself. Arrowheads in (C) and (D) shows that the space gained in both procedures is comparable. $\mathrm{E}$ : The orbital drilling in the model proposed provides an opportunity to obtain a broad corridor, thus avoiding retraction and cerebral contusion. $F$ : Access to the cranial fossa after removing the orbit in the conventional OBZ approach. In (E) and (F), it can be observed that the surgical field in the proposed and the conventional approaches is similar. OBZ: OrBitoZygomatic.

bital band as a deep limit that marks the lateral border of the superior orbital fissure. This should be carried out in the form of a "cone", in such a way that there is a translucent sheet of 


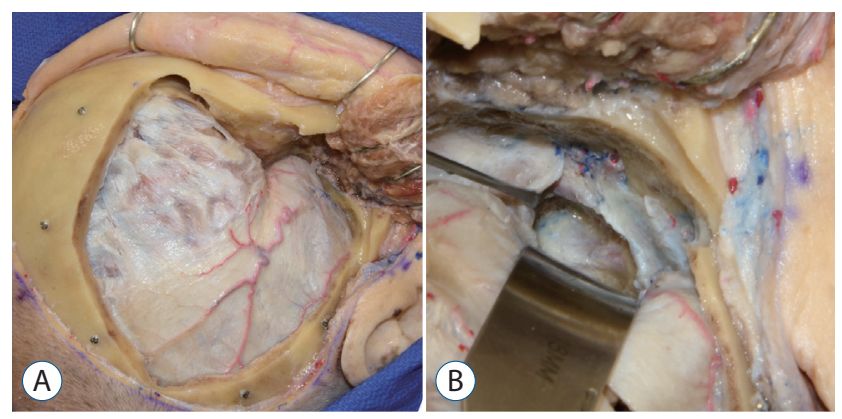

Fig. 3. Model of a complementary transzygomatic approach. A : It can be observed that by removing $3 \mathrm{~cm}$ from the zygomatic arch, the access to the infratemporal fossa is widened, which allows to increase the depth of the surgical field, the angle of vision, and reduces cerebral retraction when approaching injuries in the infratemporal fossa or the cavernous sinus. B : A tranzygomatic approach is combined with peeling of the middle fossa and a Kawase approach to access the middle fossa, with no need to remove the orbit.

bone approximately 1-mm-thick, attempting not to erode this in order to avoid herniation of the periorbital fat. For this purpose, we use cutters and round-shaped or "match-head" drillers (Fig. 2B and C). It is suggested to start with the excess bone of the orbital roof, then with the sphenoidal bone, which limits light to the middle fossa (pre-temporal zone), and finally, the bony ridge, which is localized in the orbital cone until reaching the lateral border of the superior orbital fissure (here we will find the meningeal-orbital artery and veins). By following these steps, we avoid further bleeding, because bone irrigation at this level derives mainly from the branches of the middle meningeal artery, which will only feed the bone at the base of the orbit. If necessary, anterior extradural clinoidectomy is performed. The comparison with the conventional approach (which includes orbitotomy) is observed in Fig. 2D-F.

\section{Completion of the modified OBZ approach}

Depending on the location and extent of the injury, the most suitable approach is planned, as well as the removal (or not) of the zygomatic arch (Fig. 3) and the magnitude of the temporal muscle descent (the transzygomatic approach). Nonremoval of the orbital roof using the OBZ approach concludes with this step. In the series of cases presented in this article, we performed middle fossa peeling to treat the lesions, as described below.

\section{Middle fossa peeling}

Using a microscope, the middle meningeal artery is located, which is coagulated and cut, placing bone wax into the thorny hole. If necessary, for anatomical orientation, the lateral border of the third branch of the trigeminal nerve and the intersection with the major superficial petrous nerve are identified, it being understood that underneath we find the petrous portion of the internal carotid artery.

\section{Opening the dura mater}

When lesions require dura opening, an approach can be carried out in a semi-lunar form perpendicular to the sylvian fissure with the base towards the sphenoid crest. Sometimes, the opening of the dura mater is made by an incision parallel to the sylvian fissure in such a way that, when folded back, the external dural surface adapts itself to the bone surface without the formation of folds that might obstruct the micro-neurosurgical field. By working through the natural arachnoid planes, adequate exposure can be achieved. Arachnoid dissection is performed beginning with opening the sylvian fissure to expose the internal carotid artery, the middle cerebral artery, and the anterior cerebral artery. It is advisable to perform a wide opening of the sylvian fissure in order to have a broad corridor and facilitate the visualization of neurovascular structures, without significant brain manipulation and retraction.

\section{Closure}

Closure is as important as all of the prior procedures. Once hemostasis has been verified, sufficient time must be taken to ensure hermetic closure of the dura mater. The bone fragments are usually placed and fixed in their original position. Temporal muscle is reinserted at the flange remaining for that purpose. The cutaneous flap is conventionally closed.

\section{Surgical indications of modified OBZ approach without orbital roof removal}

The OBZ approach without orbital roof removal (modified OBZ approach) provides basal exposure of the anterior and middle fossa structures. Therefore, we suggest that this approach is useful for patients with vascular lesions of the carotid bifurcation, anterior cerebral artery or bifurcation of the basilar artery, as well as lesions of the sellar and parasellar region, of the lateral wall of the cavernous sinus and in all lesions that compromise the sphenoidal wing in its medial extremity (anterior clinoid process) ${ }^{2,6,10}$. 


\section{RESULTS}

Of the 18 cases included in the study, six patients (33.3\%) were diagnosed with pituitary adenomas, six with meningiomas, two cases $(11.1 \%)$ with trigeminal schwannomas, two with sarcomas, one case (5.5\%) with fibrous dysplasia, and onecase with metastasis of a carcinoma. Age ranged from 2773 years, with an average of 46.2 years. Frequency of cases was the same for both genders (nine females and nine males). Resection degree ranged from $80-100 \%$ for all patients.

Early following complications were developed in four cases: facial edema; subgaleal hematoma; CerebroSpinal Fluid (CSF) fistula, and surgical wound dehiscence (one of each). Surgical wound dehiscence required surgical reoperation for correction. The remainder of the cases was resolved with medical treatment. The surgical procedure did not damage the cranial nerves, nor did it cause cerebral contusion, masticatory function alterations, pseudomeningocele, or cosmetic defects such as enophthalmoses or facial defects. After 36 months of follow-up, two patients died as a consequence of the natural course of the disease (one case of sarcoma and the case with metastasis). The remaining patients continue in follow-up but, to date, no complications have been reported, including five patients $(27.8 \%)$ with stable recurrences of the tumor disease (Table 1).

\section{DISCUSSION}

The minimally invasive approach is now used in all major fields of surgery, and skull base surgery is not the exception ${ }^{6,14)}$. In terms of access to skull base lesions, the minimally invasive trend has been challenged by the need to perform broader bone resections in order to minimize brain retraction but without sacrificing surgical exposure, at the expense of cosmetic and functional outcome. Therefore, achieving a balance among the extent of the osteotomy, brain tissue retraction, cosmetic and functional outcomes, and the patient's safety, involves a challenge that every neurosurgeon faces within surgical treatments of skull base lesions ${ }^{10,17,18)}$. The aim of this work was to present an approach of a conventional frontotemporo-sphenoidal (pterional) craniotomy, associated to roof

Table 1. Clinical summary of patients who underwent surgery using the OBZ approach without removal of the orbital roof

\begin{tabular}{|c|c|c|c|c|c|}
\hline Case & Gender & Age (years) & Diagnosis & Complications & $\%$ resection \\
\hline 1 & $\mathrm{~F}$ & 47 & Pituitary adenoma & Facial swelling & 100 \\
\hline 2 & $\mathrm{~F}$ & 38 & Meningioma & - & 100 \\
\hline 3 & M & 56 & Meningioma & - & 90 \\
\hline 4 & M & 29 & Pituitary adenoma & - & 90 \\
\hline 5 & M & 70 & Trigeminal schwannoma & - & 100 \\
\hline 6 & M & 45 & Meningioma & Wound dehiscence & 100 \\
\hline 7 & $\mathrm{~F}$ & 65 & Pituitary adenoma & - & 100 \\
\hline 8 & M & 43 & Pituitary adenoma & - & 90 \\
\hline 9 & $\mathrm{~F}$ & 28 & Meningioma & - & 80 \\
\hline 10 & $\mathrm{~F}$ & 34 & Trigeminal schwannoma & - & 100 \\
\hline 11 & $\mathrm{~F}$ & 39 & Sarcoma & - & 100 \\
\hline 12 & M & 41 & Meningioma & Contained CSF fistula & 100 \\
\hline 13 & $\mathrm{~F}$ & 26 & Meningioma & - & 100 \\
\hline 14 & $\mathrm{~F}$ & 73 & Pituitary adenoma & - & 100 \\
\hline 15 & M & 37 & Fibrous dysplasia & - & 100 \\
\hline 16 & M & 68 & Sarcoma & - & 90 \\
\hline 17 & M & 27 & Metastasis & - & 100 \\
\hline 18 & $\mathrm{~F}$ & 65 & Pituitary adenoma & Subgaleal hematoma & 100 \\
\hline
\end{tabular}

OBZ : OrBitoZygomatic, $F$ : female, $M$ : male, CSF : cerebrospinal fluid 
and orbital lateral wall drilling, with or without osteoplastic removal of the zygomatic arch.

\section{Surgical exposure}

The fundamental difference of our technique with respect to conventional OBZ approaches ${ }^{1,4,6,15,20-22)}$ is that we do not perform orbitotomy. Instead, we only perform orbital cone drilling until leaving a 1-mm-thick bone sheet. This allows reducing, by 3-4 mm, the visual obstacle represented by the lateral and superior orbital walls, this sufficient to enable access to the middle fossa floor ${ }^{13,17)}$. With radical drilling of the greater wing of the sphenoid bone, the medial region of the petrous temporal bone and the retropharyngeal area can be reached $^{20)}$. In addition, when the anterior part of the temporalis muscle is removed, the inferior orbital fissure can be easily accessed, which will allow the removal of bone that obstructs entry into the pathology-to-be-treated ${ }^{4)}$. Vision depth and angle are increased in horizontal and vertical planes by this approach $^{8)}$ similarly to the conventional OBZ approach, with minimal brain retraction and a simpler and faster technique $^{20,21)}$. This is supported by the work of Tanriover et al. ${ }^{22)}$, comparing the advantages and disadvantages of the one-piece vs. the two-piece OBZ approach. In their communication, the authors mentioned that, although the two-piece approach allows greater orbital roof removal, only a modest decrease is achieved with respect to the working angle, lighting, and the surgical degree-of-freedom. Meanwhile, Pritz ${ }^{17)}$ argues that there is no need to remove the orbital roof in order to access the middle fossa and the infratemporal region. It is sufficient to perform a pterional craniotomy associated with a lateral orbitotomy. In this report, we substituted the Pritz lateral orbitotomy with the drilling approach for both, roof and lateral orbital wall, obtaining similar outcomes.

Anterior extradural clinoidectomy constitutes an expansion of the approach that enhances surgical access to the skull base and contributes to safe exposure of critical neurovascular elements such as the carotid artery and the optic nerve. Consequently, less brain retraction is exerted ${ }^{16)}$.

Related to the brain-tissue retraction, we usually attempt to avoid the use of brain retractors, which can be minimized with adequate patient positioning. Adequate positioning helps the frontal lobe to precipitate naturally, discovering the orbital roof $^{15)}$. If necessary, however, it is preferred to place a brain separator for gently containing of the frontal or temporal lobe.
It is important to note that, although the orbitotomy is not as wide as that in a conventional OBZ approach, in our study no surgical brain contusions were reported.

When access to the infratemporal fossa, or to the cavernous sinus, or to a pretemporal corridor is needed, improved exposure can be afforded by an independent sectioning of $3 \mathrm{~cm}$ of the zygomatic arch. This improves the horizontal angle and avoids brain retraction ${ }^{2,4,12,21,23)}$. However, based on our experience and on what is reported by other authors, it is not mandatory in all cases to do so. We consider that carrying this out systematically in all OBZ approaches increases the surgical time and the associated morbidity, as well as facial cosmetic defects $^{2,7,8,10,17,18)}$. When required, the bone fragment remains attached to the masseter muscle, thereby facilitating surgical reconstruction and prompt recovery of the masticatory function $^{2,4,10,20,21)}$. Removal of $>3 \mathrm{~cm}$ of the zygomatic arch does not provide advantages in terms of exposure, but does increase the risk of atrophy of the muscles that contribute to chewing, and increases the risk of cosmetic defects ${ }^{2,4,18,20,21)}$.

\section{Surgical time and avoiding complications}

Reconstruction after an OBZ approach is as important as the development of the surgical technique. The more the preoperative anatomy is respected, the simpler and shorter the reconstruction will be and the better the postsurgical aesthetic outcome $e^{2,24)}$. To achieve that, it is essential to possess thorough knowledge of the anatomy and of the surgical technique, as well as to develop the ability to perform it.

Orbitotomy and the zygomatic resection of conventional approaches is a technically demanding procedure that requires time for their correct execution and repair ${ }^{8,17,24)}$. Boari et al. ${ }^{3)}$ reported that the mean time required for orbitotomy and orbital reconstruction was about half an hour in a fronto-OBZ approach. The technique that we propose has the advantage that, by not removing the orbit, its reconstruction is not necessary, in that a thin bony plate is preserved to contain the eyeball and the periorbital soft tissues. Consequently, by avoiding osteotomy and bone reconstruction, surgical time is significantly shortened ${ }^{2,8,17,20)}$.

Pulsatile enophthalmos or the development of exophthalmos has been one of the most recurrent and significant complications associated with lateral and superior orbital wall removal from the OBZ approach ${ }^{3,9,11,12,19)}$. Even in conventional approaches, it is argued that it is necessary to respect at least 
one-third of the orbital roof and to carry out an excellent anatomical reconstruction to avoid modifications in eyeball position ${ }^{6,19,20,22,25)}$. In addition, during orbitotomy, periorbital soft tissues are exposed, or frontal sinus penetration is possi$\mathrm{ble}^{2,20,22)}$. Penetrating injuries of the frontal sinus can cause considerable mucosal damage, the risk of CSF fistula, and infection, as well as time-consuming repair ${ }^{20)}$. Hence, it is important to highlight that the drilling procedure must be conducted with great care, in order to avoid the rupture of the thin bony layer that is obtained at the end of the procedure. By preserving the orbit, we have not only afforded protection for the bone, but also in terms of periorbital-tissue appearance and function, in addition to reducing the possibility of enophthalmos significantly.

Another significant aspect in functional recovery and the postsurgical aesthetic outcome is temporal muscle management. Muscle interfacial dissection allows to prevent damage of the frontotemporal branch of facial nerve that runs through adipose tissue, reducing the risk of post-surgical facial paraly$\operatorname{sis}^{1,2,4,15,25)}$. On the other hand, retrograde dissection of the deep temporalis fascia preserves the aponeurotic leaf and the neurovascular contribution, with which the problems of postoperative atrophy are smaller. It is important to emphasize avoiding the use of monopolar electrocautery, because it significantly damages blood vessels, nerves, muscle, and periosteum, which results in atrophy, fibrosis, and posterior retraction, in turn contributing to patient morbidity ${ }^{2,1524)}$.

\section{Complications rate}

The postoperative complications rate in this series is $22.2 \%$ ( $\mathrm{n}=4$ of 18). This rate is similar to that reported by other authors in conventional one-, two-, and three-piece OBZ approaches $^{2,4,24)}$. It is even lower in relation to that of other published series ${ }^{20,21)}$. None of the complications developed by our patients were conditioned by the technique of drilling or specifically by the surgical approach ${ }^{25}$. Facial edema, is an inherent complication of the technique that occurs in the majority of patients, and this was satisfactorily resolved within a few days after the procedure with rest and non-steroidal anti-inflammatory drugs ${ }^{4,25}$. The other events (subgaleal hematoma, surgical wound dehiscence, and contained CSF fistula) were satisfactorily resolved without permanent sequelae. It should be noted that there were no additional serious complications recorded, such as enophthalmos, pulsatile exophthalmos, ret- roocular pain, or cosmetic defects that have been mentioned in other series as a consequence of conventional approaches ${ }^{2,15}$. It is not recommended to use this approach in cases where tumoral lesions are over spread to the orbit.

\section{Indications of the modified OBZ approach proposed}

It is important to emphasize that the surgical technique proposed does not completely replace conventional OBZ craniotomy. Certainly, in cases where tumoral lesions involve the orbit or the periorbital soft tissues, it will be fundamental to make a partial or complete orbitototomy (medial or lateral) if necessary. The superiority of this variant over the conventional OBZ craniotomy in the cases where we suggest its application, must be determined in future comparative studies that analyze the surgical field amplitude, working angles, brain retraction degree, surgical time, and functional and cosmetic outcomes for the patients. All of these comprise variables that exert a significant impact on the choice of the most convenient technique for each case.

\section{Limitations of the study}

We recognize that the usefulness of the surgical approach that we propose in this study is based on our experience, on a small series and in a cross-sectional design. Further studies in greater samples that objectively measure the differences with respect to other variants of the OBZ approach are needed.

\section{CONCLUSION}

Based on our experience, appropriate drilling without removing the roof and lateral wall of the orbit provides a good viewing angle, similar to that achieved in conventional OBZ approaches, but with the following advantages : 1) it shortens surgical time, 2) it is technically simpler, 3) it facilitates reconstruction, 4) it minimizes surgical manipulation, 5) brain retraction is not increased, and 6) it reduces the risk of complications and cosmetic alterations. All of these advantages are in line with the basic principles of minimally invasive surgery. Nevertheless, this modified surgical technique does not completely replace the conventional OBZ approach. The final decision must be taken on the basis of surgeon preferences and a comprehensive evaluation of each particular case. 


\section{CONFLICTS OF INTEREST}

No potential conflict of interest relevant to this article was reported.

\section{INFORMED CONSENT}

Informed consent was obtained from all individual participants included in this study.

\section{References}

1. Aziz KM, Froelich SC, Cohen PL, Sanan A, Keller JT, van Loveren HR : The one-piece orbitozygomatic approach: the MacCarty burr hole and the inferior orbital fissure as keys to technique and application. Acta Neurochir (Wien) 144 : 15-24, 2002

2. Balasingam V, Noguchi A, McMenomey SO, Delashaw JB : Frontotemporal-orbitozygomatic approach. Neurosurg Q 15 : 113-121, 2005

3. Boari N, Spina A, Giudice L, Gorgoni F, Bailo M, Mortini P : Frontoorbitozygomatic approach: functional and cosmetic outcomes in a series of 169 patients. J Neurosurg 128 : 466-474, 2018

4. Campero A, Martins C, Socolovsky M, Torino R, Yasuda A, Domitrovic $L$, et al. : Three-piece orbitozygomatic approach. Neurosurgery 66(3 Suppl Operative) : E119-E120; discussion E120, 2010

5. Chaddad Neto F, Doria Netto HL, Campos Filho JM, Reghin Neto M, Silva-Costa MD, Oliveira E : Orbitozygomatic craniotomy in three pieces: tips and tricks. Arq Neuropsiquiatr 74 : 228-234, 2016

6. Cherekaev VA, Gol'bin DA, Belov Al, Radchenkov NS, Lasunin NV, Vinokurov AG : Orbitozygomatic approaches to the skull base. Zh Vopr Neirokhir Im N N Burdenko 79 : 95-101, 2015

7. Ercan S, Scerrati A, Wu P, Zhang J, Ammirati $M$ : Is less always better? Keyhole and standard subtemporal approaches: evaluation of temporal lobe retraction and surgical volume with and without zygomatic osteotomy in a cadaveric model. J Neurosurg 127 : 157-164, 2017

8. Figueiredo EG, Deshmukh P, Nakaji P, Shu EBS, Crawford N, Spetzler RF, et al. : An anatomical analysis of the mini-modified orbitozygomatic and supra-orbital approaches. J Clin Neurosci 19 : 1545-1550, 2012

9. Fujitsu K, Kuwabara $T$ : Zygomatic approach for lesions in the interpeduncular cistern. J Neurosurg 62 : 340-343, 1985

10. Galzio, RJ, Tschabitscher M, Ricci A : Orbitozygomatic Approach in Cappabianca P, Califano L, laconetta G (eds) : Cranial, Craniofacial and
Skull Base Surgery, 1st ed. Milano : Springer-Verlag, 2010, pp61-86

11. Hakuba A, Liu S, Nishimura $S:$ The orbitozygomatic infratemporal approach: a new surgical technique. Surg Neurol 26 : 271-276, 1986

12. Hakuba A, Tanaka K, Suzuki T, Nishimura S: A combined orbitozygomatic infratemporal epidural and subdural approach for lesions involving the entire cavernous sinus. J Neurosurg 71(5 Pt 1) : 699-704, 1989

13. laconetta G, Ferrer E, Galino AP, Enseñat J, de Notaris M : Frontotemporal Approach in Cappabianca P, Califano L, laconetta G (eds) : Cranial, Craniofacial and Skull Base Surgery, 1st ed. Milano : SpringerVerlag, pp39-59

14. Kim IS, Rha HK, Lee KJ, Cho KK, Park SC, Park HK, et al. : Surgical approaches to the middle cranial base tumors. J Korean Neurosurg Soc 30 : 1079-1085, 2001

15. Lemole GM Jr, Henn JS, Zabramski JM, Spetzler RF : Modifications to the orbitozygomatic approach. Technical note. J Neurosurg 99 : 924-930, 2003

16. Ota N, Tanikawa R, Miyazaki T, Miyata S, Oda J, Noda K, et al. : Surgical microanatomy of the anterior clinoid process for paraclinoid aneurysm surgery and efficient modification of extradural anterior clinoidectomy. World Neurosurg 83 : 635-643, 2015

17. Pritz $\mathrm{MB}$ : Lateral orbital rim osteotomy in the treatment of certain skull base lesions. Skull Base 12 : 1-8, 2002

18. Schwartz MS, Anderson GJ, Horgan MA, Kellogg JX, McMenomey SO, Delashaw JB Jr : Quantification of increased exposure resulting from orbital rim and orbitozygomatic osteotomy via the frontotemporal transsylvian approach. J Neurosurg 91 : 1020-1026, 1999

19. Sekhar LN, Kalia KK, Yonas H, Wright DC, Ching H : Cranial base approaches to intracranial aneurysms in the subarachnoid space. Neurosurgery 35 : 472-481; discussion 481-483, 1994

20. Taguchi Y, Tanaka K, Matsuzawa M, Sekino H : A surgical technique to avoid postoperative enophthalmos in the cranioorbital approach: technical note. J Neurosurg 85 : 514-517, 1996

21. Taguchi $Y$, Yamaguchi $Y$, Sekino $\mathrm{H}$ : Frontotemporal orbitozygomaticoalar approachfor skull surgery. J Clin Neurosci 2 : 229-234, 1995

22. Tanriover N, Ulm AJ, Rhoton AL Jr, Kawashima M, Yoshioka N, Lewis SB : One-piece versus two-piece orbitozygomatic craniotomy: quantitative and qualitative considerations. Neurosurg 58(4 Suppl 2) : ONS-229ONS-237; discussion ONS-237, 2006

23. Uttley D, Archer DJ, Marsh HT, Bell BA : Improved access to lesions of the central skull base by mobilization of the zygoma: experience with 54 cases. Neurosurgery 28 : 99-103; discussion 104, 1991

24. Youssef AS, Willard L, Downes A, Olivera R, Hall K, Agazzi S, et al. : The frontotemporal-orbitozygomatic approach: reconstructive technique and outcome. Acta Neurochir (Wien) 154 : 1275-1283, 2012

25. Zabramski JM, Kiriş T, Sankhla SK, Cabiol J, Spetzler RF : Orbitozygomatic craniotomy. Technical note. J Neurosurg 89 : 336-341, 1998 